\title{
Divergence-free Nonrenormalizable Models
}

\author{
John R. Klauder \\ Department of Physics and \\ Department of Mathematics \\ University of Florida \\ Gainesville, FL 32611
}

\begin{abstract}
A natural procedure is introduced to replace the traditional, perturbatively generated counter terms to yield a formulation of covariant, self-interacting, nonrenormalizable scalar quantum field theories that has the added virtue of exhibiting a divergence-free perturbation analysis. To achieve this desirable goal it is necessary to reexamine the meaning of the free theory about which such a perturbation takes place.
\end{abstract}

\section{Introduction}

Nonrenormalizable quantum field theories, such as $\varphi_{n}^{4}$ models, with a spacetime dimension $n \geq 5$, need nontrivial counter terms for otherwise they lead to (generalized) free theories as shown by Aizenman [1] and Fröhlich [2]. A free quantum theory has a trivial classical limit and so it can not correspond to the correct quantization of the original nontrivial classical theory. Regularized perturbation theory suggests an unending series of distinct and ever more singular counter terms which cannot be considered an acceptable solution. This situation suggests that we look elsewhere for suitable counter terms, and this paper addresses one such search.

In Sec. 2 we outline a Euclidean space lattice model for $\varphi_{n}^{4}$ models for $n \geq 5$, which includes an unconventional counter term along with the traditional terms expected in such a lattice formulation. In Sec. 3 we present the 
background for choosing this form for the model and explain the rationale for choosing the atypical counter term. Section 4 shows that general correlation functions can be suitably bounded by correlation functions at a sharp time as determined by the ground-state distribution. In Sec. 5 we take up the question of the continuum limit and study mass renormalization, coupling constant renormalization, and field strength renormalization. Here we show, thanks to the properties of our chosen counter term, that a suitable perturbation theory of the quartic interaction is divergence free. Importantly, this perturbation theory is not about the usual free theory but about a pseudofree theory, which is a model that contains the atypical counter term but does not include the quartic interaction. The reason for this divergence-free character is related to a simple idea already illustrated by idealized lattice space integrals presented in Sec. 3. Finally, Sec. 6 offers additional discussion and conclusions, and suggests a possible application of the present kind of approach to other models, most specifcally the $\varphi_{4}^{4}$ model which is perturbatively renormalizable, but generally regarded as becoming a (generalized) free theory when studied as the continuum limit of a conventional lattice formulation. While our proposal seems to be analytically challenging, there is the strong possibility that numerical Monte Carlo methods may prove useful.

\section{Overview of the Model}

\section{Preliminaries}

The present section is devoted to a presentation of the model, while the following sections discuss the motivation and analysis that has led to the present formulation. We focus on $\varphi_{n}^{4}, n \geq 5$, models formulated as Euclidean functional integrals; other models may possibly be treated by analogous procedures.

We suppose Euclidean spacetime is replaced by a periodic, hypercubic lattice with $L$ sites on an edge, $L<\infty$, and a uniform lattice spacing of $a$, $a>0$. Let the sites be labeled by $k=\left(k_{0}, k_{1}, k_{2}, \ldots, k_{s}\right)$, where $k_{j} \in \mathbb{Z}, k_{0}$ denotes the future time direction under a Wick rotation, and $s=n-1$. We denote lattice sums (and products) over all lattice points by $\Sigma_{k}$ (and $\Pi_{k}$ ), and, importantly, lattice sums (and products) over just a spatial slice at a fixed value of $k_{0}$ by $\Sigma_{k}^{\prime}$ (and $\Pi_{k}^{\prime}$ ). The total number of sites is $N=L^{n}$, while 
the number of lattice sites in a spatial slice is $N^{\prime}=L^{s}$.

In eventually taking the continuum limit we shall do so in two steps. First, we let $L \rightarrow \infty$ and $a \rightarrow 0$ together so that the full spacetime volume $V=(L a)^{n}$ remains large but finite; so too for the spatial volume $V^{\prime}=(L a)^{s}$. Second, we take the limit that both $V$ and $V^{\prime}$ diverge. In this fashion we can discuss finite spatial volumes which would have been less convenient if we had let $L \rightarrow \infty$ before taking the limit $a \rightarrow 0$.

\section{Lattice action}

Following aspects of the discussion in [3], we first introduce an important set of dimensionless constants by

$$
J_{k, l} \equiv \frac{1}{2 s+1} \delta_{k, l \in\left\{k \cup k_{n n}\right\}},
$$

where $\delta_{k, l}$ is a Kronecker delta. This notation means that an equal weight of $1 /(2 s+1)$ is given to the $2 s+1$ points in the set composed of $k$ and its $2 s$ nearest neighbors in the spatial sense only; $J_{k, l}=0$ for all other points in that spatial slice. [Specifically, we define $J_{k, l}=1 /(2 s+1)$ for the points $l=k=$ $\left(k_{0}, k_{1}, k_{2}, \ldots, k_{s}\right), l=\left(k_{0}, k_{1} \pm 1, k_{2}, \ldots, k_{s}\right), l=\left(k_{0}, k_{1}, k_{2} \pm 1, \ldots, k_{s}\right), \ldots$, $l=\left(k_{0}, k_{1}, k_{2}, \ldots, k_{s} \pm 1\right)$.] This definition implies that $\Sigma_{l}^{\prime} J_{k, l}=1$.

We next write the lattice action for the full theory, including the quartic nonlinear interaction as well as the proposed counter term, as

$$
\begin{array}{r}
I(\phi, \hbar, N)=\frac{1}{2} \sum_{k} \sum_{k^{*}}\left(\phi_{k^{*}}-\phi_{k}\right)^{2} a^{n-2}+\frac{1}{2} m_{0}^{2} \sum_{k} \phi_{k}^{2} a^{n} \\
+\lambda_{0} \sum_{k} \phi_{k}^{4} a^{n}+\frac{1}{2} \hbar^{2} \sum_{k} \mathcal{F}_{k}(\phi) a^{n}
\end{array}
$$

where $k^{*}$ denotes the $n$ nearest neighbors to $k$ in the positive sense, i.e., $k^{*} \in\left\{\left(k_{0}+1, k_{1}, \ldots, k_{s}\right), \ldots,\left(k_{0}, k_{1}, \ldots, k_{s}+1\right)\right\}$. The last term, which

represents the heart of the present procedure, is the suggested counter term and is given (with all the following sums over the spatial slice at fixed $k_{0}$ ) by

$$
\begin{aligned}
\mathcal{F}_{k}(\phi) \equiv \frac{1}{4}\left(\frac{N^{\prime}-1}{N^{\prime}}\right)^{2} a^{-2 s} \sum_{r, t}^{\prime} \frac{J_{r, k} J_{t, k} \phi_{k}^{2}}{\left[\sum_{l}^{\prime} J_{r, l} \phi_{l}^{2}\right]\left[\sum_{m}^{\prime} J_{t, m} \phi_{m}^{2}\right]} \\
-\frac{1}{2}\left(\frac{N^{\prime}-1}{N^{\prime}}\right) a^{-2 s} \sum_{t}^{\prime} \frac{J_{t, k}}{\left[\Sigma_{m}^{\prime} J_{t, m} \phi_{m}^{2}\right]} \\
+\left(\frac{N^{\prime}-1}{N^{\prime}}\right) a^{-2 s} \sum_{t}^{\prime} \frac{J_{t, k}^{2} \phi_{k}^{2}}{\left[\sum_{m}^{\prime} J_{t, m} \phi_{m}^{2}\right]^{2}} .
\end{aligned}
$$


Observe that we have included the proper dependence on $\hbar$ for the counter term implying that its contribution disappears in the classical limit in which $\hbar \rightarrow 0$. It may be noticed that each of the separate parts of the counter term scales as the inverse square of the overall field magnitude. The reason we have chosen the given counter term will be discussed in the following sections; in Sec. 6 we even show that the counter term may be considered to arise from a factor ordering ambiguity of the conventional theory.

One feature of the counter term is the fact that each term involves up to two nearest-neighbor, spatially separated lattice points. This feature is part of the regularization in the lattice formulation of the model. However, if a second-order phase transition is achieved in the continuum limit, then such a regularization should still lead to a relativistic theory in the limit.

It is important to note that as $\lambda_{0} \rightarrow 0$ and the quartic interaction is turned off, the lattice action does not pass to that of the usual free theory but to that of the free theory plus the original counter term. Such a theory has been called a pseudofree theory [4], and we shall show that the interacting theory with $\lambda_{0}>0$ exhibits a divergence-free perturbation series about the pseudofree theory. A natural argument in favor of the pseudofree theory is given in Sec. 6.

In conventional quantum field theory, counter terms are chosen to deal with the emergence of divergences; in the approach adopted in this paper, the counter term is chosen to deal with the cause of divergences. Relative to conventional treatments, therefore, it is safe to say that using the new counter term changes everything relative to what one normally expects based on the usual free theory. In particular, do not look for 'normal ordering'; instead, look for 'multiplicative renormalization'.

\section{Generating function}

One important ingredient has been left out of the lattice action, and that is the factor $Z$ representing the field strength renormalization. We introduce this factor most simply by adopting the following expression for the lattice space generating function:

$$
S(h) \equiv M_{0} \int e^{Z^{-1 / 2} \Sigma_{k} h_{k} \phi_{k} a^{n} / \hbar-I(\phi, a, N) / \hbar} \Pi_{k} d \phi_{k},
$$


where $\left\{h_{k}\right\}$ determines an appropriate test sequence, and the normalization factor $M_{0}$ ensures that $S(0)=1$. By a field rescaling, i.e., $\phi_{k} \rightarrow Z^{1 / 2} \phi_{k}$, the factor $Z$ can be removed from the source term and introduced into the lattice action; we shall have occasion to use both forms of this integral. The form of the generating function in terms of physical fields is given in Eq. (61).

The continuum limit will be taken as

$$
E\{h\} \equiv \lim _{a, L} S(h),
$$

as $a \rightarrow 0$ and $L \rightarrow \infty$ together such that, as discussed above, $C \equiv L a$ remains constant and finite. The argument of $E$ involves suitable limiting test functions $h_{k} \rightarrow h(x)$ where $k a \rightarrow x \in \mathbb{R}^{n}$. For sufficiently large $C$, it may be unnecessary to take the final limit $C \rightarrow \infty$.

\section{Rationale for Counter Term}

From the lattice action it is a simple step to write down the lattice Hamiltonian operator

$$
\begin{aligned}
\mathcal{H} \equiv & -\frac{1}{2} \hbar^{2} a^{-s} \sum_{k}{ }^{\prime} \frac{\partial^{2}}{\partial \phi_{k}^{2}}+\mathcal{V}(\phi) \\
\equiv-\frac{1}{2} \hbar^{2} a^{-s} \sum_{k}{ }^{\prime} \frac{\partial^{2}}{\partial \phi_{k}^{2}}+\mathcal{V}_{0}(\phi)+\frac{1}{2} \hbar^{2} \sum_{k}{ }^{\prime} \mathcal{F}_{k}(\phi) a^{s} & \\
=-\frac{1}{2} \hbar^{2} a^{-s} \sum_{k}{ }^{\prime} \frac{\partial^{2}}{\partial \phi_{k}^{2}}+\frac{1}{2} \sum_{k}^{\prime} \sum_{k^{*}}^{\prime}\left(\phi_{k^{*}}-\phi_{k}\right)^{2} a^{s-2}+\frac{1}{2} m_{0}^{2} \sum_{k}{ }^{\prime} \phi_{k}^{2} a^{s} & \quad+\lambda_{0} \sum_{k}{ }^{\prime} \phi_{k}^{4} a^{s}+\frac{1}{2} \hbar^{2} \sum_{k}{ }^{\prime} \mathcal{F}_{k}(\phi) a^{s}-E_{0} .
\end{aligned}
$$

In addition, we introduce the ground state $\Psi(\phi)$ for this Hamiltonian operator. The constant $E_{0}$ is chosen so that $\Psi(\phi)$ satisfies the Schrödinger equation

$$
\mathcal{H} \Psi(\phi)=0,
$$

which implies that the Hamiltonian operator can also be written as

$$
\mathcal{H}=-\frac{\hbar^{2}}{2} a^{-s} \sum_{k}{ }^{\prime} \frac{\partial^{2}}{\partial \phi_{k}^{2}}+\frac{\hbar^{2}}{2} a^{-s} \sum_{k}{ }^{\prime} \frac{1}{\Psi(\phi)} \frac{\partial^{2} \Psi(\phi)}{\partial \phi_{k}^{2}} .
$$


Since the ground state $\Psi(\phi)$ does not vanish, it can be written in the generic form

$$
\Psi(\phi)=\frac{e^{-U(\phi, a, \hbar) / 2}}{D(\phi)},
$$

and thus (using the abbreviation $X,_{k} \equiv \partial X / \partial \phi_{k}$ and the spatial summation convention)

$$
\begin{aligned}
\mathcal{V}(\phi) & =\frac{1}{2} \hbar^{2} a^{-s} D e^{U / 2}\left[D^{-1} e^{-U / 2}\right]_{, k k} \\
& =\frac{1}{2} \hbar^{2} a^{-s}\left[\frac{1}{4} U^{2},_{k}-\frac{1}{2} U_{, k k}+D^{-1} U_{, k} D_{, k}+2 D^{-2} D_{k}^{2}-D^{-1} D_{, k k}\right] .
\end{aligned}
$$

We insist that the atypical counter term $\mathcal{F}_{k}(\phi)$ is determined by the denominator $D$ alone by requiring that

$$
\frac{1}{2} \hbar^{2} \sum_{k}{ }^{\prime} \mathcal{F}_{k}(\phi) a^{s} \equiv \frac{1}{2} \hbar^{2} a^{-s} D D^{-1}{ }_{, k k}=\frac{1}{2} \hbar^{2} a^{-s}\left[2 D^{-2} D^{2}{ }_{, k}-D^{-1} D,,_{k k}\right] .
$$

There are multiple solutions to this equation which lead to ground state functions that are locally square integrable near the origin in field space. However, the only solution consistent with a nowhere vanishing ground state is given (up to an overall factor) by

$$
D(\phi)=\Pi_{k}^{\prime}\left[\Sigma_{l}^{\prime} J_{k, l} \phi_{l}^{2}\right]^{\left(N^{\prime}-1\right) / 4 N^{\prime}} .
$$

In point of fact, $D$ was chosen first, and the counter term was then derived from $D$ by this very differential equation. Why we have chosen this specific form for $D$ is discussed below.

The ground state $\Psi(\phi)$ leads to the probability density

$$
\Psi(\phi)^{2} \equiv K \frac{e^{-U(\phi, a, \hbar)}}{\Pi_{k}^{\prime}\left[\Sigma_{l}^{\prime} J_{k, l} \phi_{l}^{2}\right]^{\left(N^{\prime}-1\right) / 2 N^{\prime}}},
$$

where $K$ accounts for normalization of this expression given the additional assumption that $U(0, a, \hbar)=0$. The normalization integral itself then reads

$$
K \int \frac{e^{-U(\phi, a, \hbar)}}{\Pi_{k}^{\prime}\left[\Sigma_{l}^{\prime} J_{k, l} \phi_{l}^{2}\right]^{\left(N^{\prime}-1\right) / 2 N^{\prime}}} \Pi_{k}^{\prime} d \phi_{k}=1 .
$$

Before commenting on this integral further, we wish to discuss several simpler integrals. 


\section{A discussion of many-dimensional integrals}

Consider the family of Gaussian integrals given (at some fixed $k_{0}$ ) by

$$
I_{G}(2 p) \equiv \int\left[\Sigma_{k}^{\prime} \phi_{k}^{2}\right]^{p} e^{-A \Sigma_{k}^{\prime} \phi_{k}^{2}} \Pi_{k}^{\prime} d \phi_{k},
$$

where $p \in\{0,1,2,3, \ldots\}$, and we assume that $A$ is of 'normal size', e.g., $0.1<A<10$. Although these integrals can be evaluated explicitly, we prefer to study the qualitative behavior of such integrals for large values of $N^{\prime}$, i.e., when $N^{\prime} \gg 1$. For this purpose it is highly instructive to introduce hyper-spherical coordinates $[5,6]$ defined by

$$
\begin{gathered}
\phi_{k} \equiv \kappa \eta_{k}, \quad 0 \leq \kappa<\infty, \quad-1 \leq \eta_{k} \leq 1, \\
\Sigma_{k}^{\prime} \eta_{k}^{2} \equiv 1, \quad \Sigma_{k}^{\prime} \phi_{k}^{2} \equiv \kappa^{2} .
\end{gathered}
$$

Here $\kappa$ acts as a hyper-radius field variable and the $\left\{\eta_{k}\right\}$ variables constitute an $N^{\prime}$-dimensional direction field. Note well that $\kappa \equiv \sqrt{\Sigma_{k}^{\prime} \phi_{k}^{2}}$ is the 'radius' of all the field variables in a given spatial slice of the lattice at some fixed $k_{0}$. In terms of these variables, it follows that

$$
I_{G}(2 p)=2 \int\left[\kappa^{2}\right]^{p} e^{-A \kappa^{2}} \kappa^{N^{\prime}-1} d \kappa \delta\left(1-\Sigma_{k}^{\prime} \eta_{k}^{2}\right) \Pi_{k}^{\prime} d \eta_{k} .
$$

Observe that the integrand depends on the radius $\kappa$, but it does not depend on the angular variables $\left\{\eta_{k}\right\}$. For very large $N^{\prime}$, the integral over $\kappa$ can be studied by steepest descent methods. To leading order, the integrand is narrowly peaked at values of $\kappa \simeq\left(N^{\prime} / 2 A\right)^{1 / 2}$, namely at large values of $\kappa$. As a consequence, for each value of $A$, the integrand is supported on a disjoint set of $\kappa$ as $N^{\prime} \rightarrow \infty$. This well-known fact [7] leads to divergences in perturbation calculations. For example, let us study

$$
I_{G}^{\star}(2)=\int\left[\Sigma_{k}^{\prime} \phi_{k}^{2}\right] e^{-A^{\star} \Sigma_{k}^{\prime} \phi_{k}^{2}} \Pi_{k}^{\prime} d \phi_{k},
$$

which is the same type of Gaussian integral for a different value of $A$. For this study, we introduce the perturbation series

$$
I_{G}^{\star}(2)=I_{G}(2)-\Delta A I_{G}(4)+\frac{1}{2}(\Delta A)^{2} I_{G}(6)-\cdots,
$$


where $\Delta A \equiv A^{\star}-A$. Since $I_{G}(2 p) / I_{G}(2)=O\left(N^{\prime(p-1)}\right)$, this series exhibits divergences as $N^{\prime} \rightarrow \infty$. It is not difficult to convince oneself that such divergences are quite analogous to those that appear in quantum field theory; see [5] for further examples of this sort.

If the factor $\kappa^{\left(N^{\prime}-1\right)}$ is removed from $I_{G}(2 p)$, we are led to consider

$$
I_{G}^{\prime}(2 p)=2 \int\left[\kappa^{2}\right]^{p} e^{-A \kappa^{2}} d \kappa \delta\left(1-\Sigma_{k}^{\prime} \eta_{k}^{2}\right) \Pi_{k}^{\prime} d \eta_{k}
$$

Now, the integrand is broadly supported and no longer favors large $\kappa$ values. Consequently, a perturbation series evaluation of

$$
\begin{aligned}
I_{G}^{\prime \star}(2) & =2 \int \kappa^{2} e^{-A^{\star} \kappa^{2}} d \kappa \delta\left(1-\Sigma_{k}^{\prime} \eta_{k}^{2}\right) \Pi_{k}^{\prime} d \eta_{k} \\
& =I_{G}^{\prime}(2)-\Delta A I_{G}^{\prime}(4)+\frac{1}{2}(\Delta A)^{2} I_{G}^{\prime}(6)-\cdots
\end{aligned}
$$

exhibits no divergences since $I_{G}^{\prime}(2 p) / I_{G}^{\prime}(2)=O\left(N^{\prime 0}\right)$.

Unlike the original integrals over $\kappa$, integrals over the $\left\{\eta_{k}\right\}$ direction field variables cannot diverge under normal circumstances since each variable satisfies $-1 \leq \eta_{k} \leq 1$. We will encounter such variables in the denominator of (14); however, the form of that denominator has been specifically chosen to ensure that such integrals converge near zero for all $N^{\prime}<\infty$.

\section{Relevance for the ground-state distribution}

The normalization integral for the ground-state distribution, Eq. (14), expressed in terms of hyper-spherical coordinates, becomes

$$
2 K \int \frac{e^{-U(\kappa \eta, a, \hbar)}}{\Pi_{k}^{\prime}\left[\Sigma_{l}^{\prime} J_{k, l} \eta_{l}^{2}\right]^{\left(N^{\prime}-1\right) / 2 N^{\prime}}} d \kappa \delta\left(1-\Sigma_{k}^{\prime} \eta_{k}^{2}\right) \Pi_{k}^{\prime} d \eta_{k}=1
$$

Note well that the absence of the factor $\kappa^{\left(N^{\prime}-1\right)}$ in this expression is a direct result of the counter term in the lattice Hamiltonian, which in turn gave rise to the denominator factor $D$ in the ground state $\Psi(\phi)$. Just like the elementary examples in which $\kappa^{\left(N^{\prime}-1\right)}$ was artificially removed, there is no peaking of the integrand in $\kappa$ due to that factor. For integrals such as

$$
K \int\left[\Sigma_{k}^{\prime} \phi_{k}^{2}\right]^{p} \frac{e^{-U(\phi, a, \hbar)}}{\Pi_{k}^{\prime}\left[\Sigma_{l}^{\prime} J_{k, l} \phi_{l}^{2}\right]^{\left(N^{\prime}-1\right) / 2 N^{\prime}}} \Pi_{k}^{\prime} d \phi_{k}
$$


it is clear that the $\kappa$-dependence of the integrand is most likely spread rather broadly; this conclusion would be false if the factor $\kappa^{\left(N^{\prime}-1\right)}$ had not been canceled by part of the term $D^{2}$.

\section{Correlation Functions and their Bounds}

In this section, following [3], we wish to show that the full spacetime correlation functions can be controlled by their sharp-time behavior along with a suitable choice of test sequences.

Let the notation

$$
\phi_{u} \equiv \Sigma_{k} u_{k} \phi_{k} a^{n}
$$

denote the full spacetime summation over all lattice sites where $\left\{u_{k}\right\}$ denotes a suitable test sequence. We also separate out the temporal part of this sum in the manner

$$
\phi_{u} \equiv \Sigma_{k_{0}} a \phi_{u^{\prime}} \equiv \Sigma_{k_{0}} a \Sigma_{k}^{\prime} u_{k} \phi_{k} a^{s}
$$

Observe that the notation $\phi_{u^{\prime}}$ (with the prime) implies a summation only over the spatial lattice points for a fixed (and implicit) value of the temporal lattice value $k_{0}$.

Let the notation $\langle(\cdot)\rangle$ denote full spacetime averages with respect to the field distribution determined by the lattice action, and then let us consider full spacetime correlation functions such as

$$
\left\langle\phi_{u^{(1)}} \phi_{u^{(2)}} \cdots \phi_{u^{(2 q)}}\right\rangle=\Sigma_{k_{0}^{(1)}, k_{0}^{(2)}, \ldots, k_{0}^{(2 q)}} a^{2 q}\left\langle\phi_{u^{\prime(1)}} \phi_{u^{(2)}} \cdots \phi_{u^{\prime(2 q)}}\right\rangle,
$$

where $q \geq 1$ and the expectation on the right-hand side is over products of fixed-time summed fields, $\phi_{u^{\prime}}$, for possibly different times, which are then summed over their separate times. All odd correlation functions are assumed to vanish, and furthermore, $\langle 1\rangle=1$ in this normalized spacetime lattice field distribution. It is also clear that

$$
\left|\left\langle\phi_{u^{(1)}} \phi_{u^{(2)}} \cdots \phi_{u^{(2 q)}}\right\rangle\right| \leq \Sigma_{k_{0}^{(1)}, k_{0}^{(2)}, \ldots, k_{0}^{(2 q)}} a^{2 q}\left|\left\langle\phi_{u^{\prime(1)}} \phi_{u^{\prime(2)}} \cdots \phi_{u^{(2 q)}}\right\rangle\right| .
$$

At this point we turn our attention toward the spatial sums alone. 
We appeal to straightforward inequalities of the general form

$$
\langle A B\rangle^{2} \leq\left\langle A^{2}\right\rangle\left\langle B^{2}\right\rangle
$$

In particular, it follows that

$$
\left\langle\phi_{u^{\prime}(1)} \phi_{u^{\prime}(2)} \phi_{u^{\prime}(3)} \phi_{u^{\prime}(4)}\right\rangle^{2} \leq\left\langle\phi_{u^{\prime(1)}}^{2} \phi_{u^{\prime(2)}}^{2}\right\rangle\left\langle\phi_{u^{\prime(3)}}^{2} \phi_{u^{\prime(4)}}^{2}\right\rangle
$$

and, in turn, that

$$
\begin{aligned}
\left\langle\phi_{u^{\prime}(1)} \phi_{u^{\prime}(2)} \phi_{u^{\prime}(3)} \phi_{u^{\prime}(4)}\right\rangle^{4} & \leq\left\langle\phi_{u^{\prime(1)}}^{2} \phi_{u^{\prime}(2)}^{2}\right\rangle^{2}\left\langle\phi_{u^{\prime}(3)}^{2} \phi_{u^{\prime(4)}}^{2}\right\rangle^{2} \\
& \leq\left\langle\phi_{u^{\prime(1)}}^{4}\right\rangle\left\langle\phi_{u^{\prime(2)}}^{4}\right\rangle\left\langle\phi_{u^{\prime(3)}}^{4}\right\rangle\left\langle\phi_{u^{\prime(4)}}^{4}\right\rangle .
\end{aligned}
$$

By a similar argument, it follows that

$$
\left|\left\langle\phi_{u^{\prime}(1)} \phi_{u^{\prime(2)}} \cdots \phi_{u^{\prime(2 q)}}\right\rangle\right| \leq \Pi_{j=1}^{2 q}\left[\left\langle\phi_{u^{\prime(j)}}^{2 q}\right\rangle\right]^{1 / 2 q}
$$

which has bounded any particular mixture of spatial correlation functions at possibly different times, by a suitable product of higher-power expectations each of which involves field values ranging over a spatial level, all at a single fixed lattice time. By time translation invariance of the various single time correlation functions we can assert that

$$
\left\langle\phi_{u^{\prime(j)}}^{2 r}\right\rangle
$$

which is defined at time $k_{0}^{(j)}$, is actually independent of the time and, therefore, the result could be calculated at any fixed time. In particular, we can express such correlation functions as

$$
\left\langle\phi_{u^{\prime}}^{2 q}\right\rangle=\int \phi_{u^{\prime}}^{2 q} \Psi(\phi)^{2} \Pi_{k}^{\prime} d \phi_{k} .
$$

Thus we see that a bound on full spacetime correlation functions may be given in terms of sharp-time correlation functions in the ground-state distribution.

\section{The Continuum Limit}

Before focusing on the limit $a \rightarrow 0$ and $L \rightarrow \infty$, let us note some important facts about ground-state averages of the direction field variables $\left\{\eta_{k}\right\}$. First, 
we assume that such averages have two important symmetries: (1) averages of an odd number of $\eta_{k}$ variables vanish, i.e.,

$$
\left\langle\eta_{k_{1}} \cdots \eta_{k_{2 p+1}}\right\rangle=0
$$

and (2) such averages are invariant under any spacetime translation, i.e.,

$$
\left\langle\eta_{k_{1}} \cdots \eta_{k_{2 p}}\right\rangle=\left\langle\eta_{k_{1}+l} \cdots \eta_{k_{2 p}+l}\right\rangle
$$

for any $l \in \mathbb{Z}^{n}$ due to a similar translational invariance of the lattice Hamiltonian. Second, we note that for any ground-state distribution, it is necessary that $\left\langle\eta_{k}^{2}\right\rangle=1 / N^{\prime}$ for the simple reason that $\Sigma_{k}^{\prime} \eta_{k}^{2}=1$. Hence, $\left|\left\langle\eta_{k} \eta_{l}\right\rangle\right| \leq 1 / N^{\prime}$ as follows from the Schwarz inequality. Since $\left\langle\left[\Sigma_{k}^{\prime} \eta_{k}^{2}\right]^{2}\right\rangle=1$, it follows that $\left\langle\eta_{k}^{2} \eta_{l}^{2}\right\rangle=O\left(1 / N^{\prime 2}\right)$. Indeed, similar arguments show that for any groundstate distribution

$$
\left\langle\eta_{k_{1}} \cdots \eta_{k_{2 p}}\right\rangle=O\left(1 / N^{\prime p}\right)
$$

which will be useful in the sequel.

Next, we choose to study the pseudofree model, namely, when the coupling constant $\lambda_{0}=0$. This is as close as we can get to the free model itself. Unfortunately, we cannot solve Eq. (10) when $\mathcal{V}$ has the desired form. The best we can do is choose a form for $U(\phi, a, \hbar)$ in $(9)$ that leads to an approximate form of the pseudofree model. In particular, we choose

$$
U(\phi, a, \hbar)=(1 / \hbar) \Sigma_{k, l}^{\prime} \phi_{k} A_{k-l} \phi_{l} a^{2 s} .
$$

This expression is taken to be the form for $U$ for the free model as if there was no counter term and consequently $D$ was replaced by 1 . This ensures us that the potential $\mathcal{V}(\phi)$ that follows from (10) agrees with the desired free model to leading order in $\hbar$. Specifically, with the given choice for $U$ and $D$, it follows that

$$
\begin{aligned}
\mathcal{V}(\phi)= & \frac{1}{2} \Sigma_{k, l, m}^{\prime} \phi_{k} A_{k-l} A_{l-m} \phi_{m} a^{3 s}-\frac{1}{2} \hbar A_{0} N^{\prime} a^{s}+\frac{1}{2} \hbar^{2} \Sigma_{k}^{\prime} \mathcal{F}_{k}(\phi) a^{s} \\
& +\hbar\left[\left(N^{\prime}-1\right) / 4 N^{\prime}\right] \Sigma_{k, r, m}^{\prime} J_{r, k} \phi_{k} A_{k-m} \phi_{m} /\left[\Sigma_{l}^{\prime} J_{r, l} \phi_{l}^{2}\right] a^{2 s}
\end{aligned}
$$

We choose the matrix $A_{k-l}$ so that the first term in (38) yields the desired gradient and mass terms in the Hamiltonian expression (6). In particular, we note that to match the quadratic, spatial lattice-derivative terms in the 
Hamiltonian (typically the most singular of the quadratic terms), we can do so by choosing the elements of the matrix $A_{k-l}=O\left(a^{-(s+1)}\right)$. Although this leads to only an approximate form for the pseudofree model, it is sufficient for our limited purpose at present, namely, to determine the field strength renormalization constant $Z$.

\section{Field strength renormalization}

We now take up the question of the sharp-time averages given by

$$
\int Z^{-p}\left[\Sigma_{k}^{\prime} h_{k} \phi_{k} a^{s}\right]^{2 p} \Psi(\phi)^{2} \Pi_{k}^{\prime} d \phi_{k},
$$

where $Z$ denotes the field strength renormalization factor and $\left\{h_{k}\right\}$ represents a suitable spatial test sequence. These are exactly the kinds of expression that should become well behaved in the continuum limit for a proper choice of $Z$. Thus, we are led to consider

$$
\begin{aligned}
& K \int Z^{-p}\left[\Sigma_{k}^{\prime} h_{k} \phi_{k} a^{s}\right]^{2 p} \frac{e^{-\Sigma_{k, l}^{\prime} \phi_{k} A_{k-l} \phi_{l} a^{2 s} / \hbar}}{\Pi_{k}^{\prime}\left[\Sigma_{l}^{\prime} J_{k, l} \phi_{l}^{2}\right]^{\left(N^{\prime}-1\right) / 2 N^{\prime}}} \Pi_{k}^{\prime} d \phi_{k} \\
& =2 K \int Z^{-p} \kappa^{2 p}\left[\Sigma_{k}^{\prime} h_{k} \eta_{k} a^{s}\right]^{2 p} \frac{e^{-\kappa^{2} \Sigma_{k, l}^{\prime} \eta_{k} A_{k-l} \eta_{l} a^{2 s} / \hbar}}{\Pi_{k}^{\prime}\left[\Sigma_{l}^{\prime} J_{k, l} \eta_{l}^{2}\right]^{\left(N^{\prime}-1\right) / 2 N^{\prime}}} d \kappa \delta\left(1-\Sigma_{k}^{\prime} \eta_{k}^{2}\right) \Pi_{k}^{\prime} d \eta_{k} .
\end{aligned}
$$

Our goal is to use this integral to determine a value for the field strength renormalization constant $Z$. To estimate this integral we first replace two factors with $\eta$ variables by their appropriate averages. In particular, the expression in the exponent is estimated by

$$
\kappa^{2} \Sigma_{k, l}^{\prime} \eta_{k} A_{k-l} \eta_{l} a^{2 s} \simeq \kappa^{2} \Sigma_{k, l}^{\prime} N^{\prime-1} A_{k-l} a^{2 s} \propto \kappa^{2} N^{\prime} a^{2 s} a^{-(s+1)},
$$

and the expression in the integrand is estimated by

$$
\left[\Sigma_{k}^{\prime} h_{k} \eta_{k} a^{s}\right]^{2 p} \simeq N^{\prime-p}\left[\Sigma_{k}^{\prime} h_{k} a^{s}\right]^{2 p} .
$$

The integral over $\kappa$ is then estimated by first rescaling the variable $\kappa^{2} \rightarrow$ $\kappa^{2} /\left(N^{\prime} a^{s-1}\right)$, which then leads to an overall integral-estimate proportional to

$$
Z^{-p}\left[N^{\prime} a^{s-1}\right]^{-p} N^{\prime-p}\left[\Sigma_{k}^{\prime} h_{k} a^{s}\right]^{2 p} .
$$


Finally, for this result to be meaningful in the continuum limit, we are led to choose $Z=N^{\prime-2} a^{-(s-1)}$. However, $Z$ must be dimensionless, so we introduce a fixed positive quantity $q$ with dimensions of an inverse length, which allows us to set

$$
Z=N^{\prime-2}(q a)^{-(s-1)} .
$$

This is a fundamental and important relation in our analysis.

\section{Mass renormalization}

With notation where $\langle(\cdot)\rangle$ denotes a full spacetime lattice-space average based on the lattice action for the pseudofree theory, an expansion of the mass term leads to a series of terms of the form

$$
\left\langle\left[m_{0}^{2} \Sigma_{k} \phi_{k}^{2} a^{n}\right]^{p}\right\rangle,
$$

which in turn can be expressed as

$$
m_{0}^{2 p} \Sigma_{k_{0}^{(1)}, k_{0}^{(2)}, \ldots, k_{0}^{(p)}} a^{p}\left\langle\left[\Sigma_{k^{(1)}}^{\prime} \phi_{k^{(1)}}^{2} a^{s}\right]\left[\Sigma_{k^{(2)}}^{\prime} \phi_{k^{(2)}}^{2} a^{s}\right] \cdots\left[\Sigma_{k^{(p)}}^{\prime} \phi_{k^{(p)}}^{2} a^{s}\right]\right\rangle .
$$

Based on the inequality

$$
\left\langle\Pi_{j=1}^{p} A_{j}\right\rangle \leq \Pi_{j=1}^{p}\left\langle A_{j}^{p}\right\rangle^{1 / p},
$$

valid when $A_{j} \geq 0$ for all $j$, it follows that

$$
\begin{aligned}
& \left\langle\left[m_{0}^{2} \Sigma_{k} \phi_{k}^{2} a^{n}\right]^{p}\right\rangle \leq m_{0}^{2 p} \Sigma_{k_{0}^{(1)}, k_{0}^{(2)}, \ldots, k_{0}^{(p)} a^{p}} \\
& \quad \times\left\{\left\langle\left[\Sigma_{k^{(1)}}^{\prime} \phi_{k^{(1)}}^{2} a^{s}\right]^{p}\right\rangle\left\langle\left[\Sigma_{k^{(2)}}^{\prime} \phi_{k^{(2)}}^{2} a^{s}\right]^{p}\right\rangle \cdots\left\langle\left[\Sigma_{k^{(p)}}^{\prime} \phi_{k^{(p)}}^{2} a^{s}\right]^{p}\right\rangle\right\}^{1 / p} .
\end{aligned}
$$

This leads us to consider

$$
\begin{gathered}
\left\langle\left[m_{0}^{2} \Sigma_{k}^{\prime} \phi_{k}^{2} a^{s}\right]^{p}\right\rangle=2 m_{0}^{2 p} a^{s p} K \int \kappa^{2 p} \frac{e^{-\kappa^{2} \Sigma_{k, l}^{\prime} \eta_{k} A_{k-l} \eta_{l} a^{2 s}}}{\Pi_{k}\left[\Sigma_{l}^{\prime} J_{k, l} \eta_{l}^{2}\right]^{\left(N^{\prime}-1\right) / 2 N^{\prime}}} d \kappa \\
\times \delta\left(1-\Sigma_{k}^{\prime} \eta_{k}^{2}\right) \Pi d \eta_{k},
\end{gathered}
$$

which, in the manner used previously, can be estimated as

$$
\left\langle\left[m_{0}^{2} \Sigma_{k}^{\prime} \phi_{k}^{2} a^{s}\right]^{p}\right\rangle \propto \frac{m_{0}^{2 p} a^{s p}}{\left[N^{\prime} a^{(s-1)}\right]^{p}} .
$$


To make sense in the continuum limit, this leads us to identify

$$
m_{0}^{2}=N^{\prime}(q a)^{-1} m^{2},
$$

with $m^{2}$ being the physical mass. Moreover, it is noteworthy that

$$
Z m_{0}^{2}=\left[N^{\prime-2}(q a)^{-(s-1)}\right]\left[N^{\prime}(q a)^{-1}\right] m^{2}=\left[N^{\prime}(q a)^{s}\right]^{-1} m^{2},
$$

which for a finite spatial volume $V^{\prime}=N^{\prime} a^{s}$ leads to a finite nonzero result for $Z m_{0}^{2}$.

\section{Coupling constant renormalization}

We repeat the previous calculation for an expansion of the quartic interaction term about the pseudofree theory. This leads us to consider terms of the form

$$
\left\langle\left[\lambda_{0} \Sigma_{k} \phi_{k}^{4} a^{n}\right]^{p}\right\rangle,
$$

which in turn can be expressed as

$$
\lambda_{0}^{p} \Sigma_{k_{0}^{(1)}, k_{0}^{(2)}, \ldots, k_{0}^{(p)}} a^{p}\left\langle\left[\Sigma_{k^{(1)}}^{\prime} \phi_{k^{(1)}}^{4} a^{s}\right]\left[\Sigma_{k^{(2)}}^{\prime} \phi_{k^{(2)}}^{4} a^{s}\right] \cdots\left[\Sigma_{k^{(p)}}^{\prime} \phi_{k^{(p)}}^{4} a^{s}\right]\right\rangle
$$

and bounded by

$$
\begin{aligned}
& \left\langle\left[\lambda_{0} \Sigma_{k} \phi_{k}^{4} a^{n}\right]^{p}\right\rangle \leq \lambda_{0}^{p} \Sigma_{k_{0}^{(1)}, k_{0}^{(2)}, \ldots, k_{0}^{(p)}} a^{p} \\
& \quad \times\left\{\left\langle\left[\Sigma_{k^{(1)}}^{\prime} \phi_{k^{(1)}}^{4} a^{s}\right]^{p}\right\rangle\left\langle\left[\Sigma_{k^{(2)}}^{\prime} \phi_{k^{(2)}}^{4} a^{s}\right]^{p}\right\rangle \cdots\left\langle\left[\Sigma_{k^{(p)}}^{\prime} \phi_{k^{(p)}}^{4} a^{s}\right]^{p}\right\rangle\right\}^{1 / p} .
\end{aligned}
$$

This leads us to consider

$$
\begin{gathered}
\left\langle\left[\lambda_{0} \Sigma_{k}^{\prime} \phi_{k}^{4} a^{s}\right]^{p}\right\rangle=2 \lambda_{0}^{p} a^{s p} K \int \kappa^{4 p}\left[\Sigma_{k}^{\prime} \eta_{k}^{4}\right]^{p} \frac{e^{-\kappa^{2} \Sigma_{k, l}^{\prime} \eta_{k} A_{k-l} \eta_{l} a^{2 s}}}{\Pi_{k} \mid\left[\Sigma_{l}^{\prime} J_{k, l} \eta_{l}^{2}\right]^{\left(N^{\prime}-1\right) / 2 N^{\prime}}} \\
\times d \kappa \delta\left(1-\Sigma_{k}^{\prime} \eta_{k}^{2}\right) \Pi d \eta_{k},
\end{gathered}
$$

which, in the manner used previously, can be estimated as

$$
\left\langle\left[\lambda_{0} \Sigma_{k}^{\prime} \phi_{k}^{4} a^{s}\right]^{p}\right\rangle \propto \frac{\lambda_{0}^{p} N^{\prime-p} a^{s p}}{\left[N^{\prime} a^{(s-1)}\right]^{2 p}},
$$

and to make sense in the continuum limit leads us to identify

$$
\lambda_{0}=N^{\prime 3}(q a)^{s-2} \lambda,
$$

with $\lambda$ being the physical coupling constant. Moreover, it is noteworthy that

$$
Z^{2} \lambda_{0}=\left[N^{\prime-4}(q a)^{-2(s-1)}\right]\left[N^{\prime 3}(q a)^{s-2}\right] \lambda=\left[N^{\prime}(q a)^{s}\right]^{-1} \lambda,
$$

which for a finite spatial volume $V^{\prime}=N^{\prime} a^{s}$ leads to a finite nonzero result for $Z^{2} \lambda_{0}$. 


\section{Physical version of the generating function}

Based on the previous analysis we are led to reformulate the expression for the lattice space generating function (4). We first make a change of integration variables such that $\phi_{k} \rightarrow Z^{1 / 2} \phi_{k}$ leading to the expression

$$
S(h) \equiv M \int e^{\Sigma_{k} h_{k} \phi_{k} a^{n} / \hbar-I\left(Z^{1 / 2} \phi, a, N\right) / \hbar} \Pi_{k} d \phi_{k},
$$

where any constant Jacobian factor has been absorbed into a change of the overall normalization factor from $M_{0}$ to $M$. Finally, we introduce the explicit form for the lattice action from (2) into (60) to yield

$$
\begin{aligned}
S(h)= & M \int \exp \left\{\Sigma h_{k} \phi_{k} a^{n} / \hbar\right. \\
& -\frac{1}{2}\left[N^{\prime 2}(q a)^{(s-1)}\right]^{-1} \sum_{k} \sum_{k^{*}}\left(\phi_{k^{*}}-\phi_{k}\right)^{2} a^{n-2} / \hbar \\
& -\frac{1}{2}\left[N^{\prime}(q a)^{s}\right]^{-1} m^{2} \sum_{k} \phi_{k}^{2} a^{n} / \hbar-\left[N^{\prime}(q a)^{s}\right]^{-1} \lambda \sum_{k} \phi_{k}^{4} a^{n} / \hbar \\
& \left.-\frac{1}{2} \hbar^{2}\left[N^{\prime 2}(q a)^{(s-1)}\right] \sum_{k} \mathcal{F}_{k}(\phi) a^{n} / \hbar\right\} \Pi_{k} d \phi_{k} .
\end{aligned}
$$

This expression contains a formulation of the lattice space generating function expressed in terms of physical fields and constants.

\section{Commentary}

In our final expression above there are several noteworthy points to be made. In a finite spatial volume $V^{\prime}=N^{\prime} a^{s}$ - which due to our hypercubic assumption for spacetime implies a finite spacetime volume $V=N a^{n}$ - the coefficients of the physical mass $m$ and the physical coupling constant $\lambda$ are both finite and nonzero. We have shown earlier in this section that perturbation in both the quadratic mass term and the quartic nonlinear action leads to a series which is term-by-term finite when perturbed about the pseudofree theory. If the finite spatial volume is taken large enough (e.g., Milky Way sized), then a divergence-free perturbation series is established. In other words, the introduction of the unusual counter term has resolved any issues with typical ultraviolet divergences (and it is noteworthy that it has not been necessary to maintain an ultraviolet cutoff to achieve this goal).

[Remark: Any theory exhibits infinite volume divergences for questions of a stationary nature. For example, such divergences even arise already when 
$n=1$ and we deal with time alone, as for example with a conventional, stationary Ornstein-Uhlenbeck (O-U) process $U(t) \equiv 2^{-1 / 2} e^{-t} W\left(e^{2 t}\right),-\infty<$ $t<\infty$, where $W(\tau), 0 \leq \tau<\infty$, denotes a standard, Gaussian, Wiener process for which $W(0)=0, \mathbf{E}(W(\tau))=0$, and $\mathbf{E}\left(W\left(\tau_{1}\right) W\left(\tau_{2}\right)\right)=\min \left(\tau_{1}, \tau_{2}\right)$, where $\mathbf{E}$ denotes ensemble average. Although the $\mathrm{O}-\mathrm{U}$ paths are concentrated on bounded, continuous paths, it nevertheless follows that

$$
\mathbf{E}\left(\int_{-\infty}^{\infty} U(t)^{2} d t\right)=\infty
$$

due to the stationarity of the process.]

Unlike the quadratic mass and quartic interaction terms, the coefficients of the derivative terms and the inverse-square field counter term are inverse to one another and do not have finite nonzero limits when $L \rightarrow \infty$ and $a \rightarrow 0$ such that the spatial volume $V^{\prime}=(L a)^{s}$ is finite. This aspect is not unexpected since (i) the coefficient of the counter term is intimately linked to that of the derivative term so that the field power that appears in the denominator factor $D^{2}$ in the ground state distribution is $\left(N^{\prime}-1\right) / 2 N^{\prime}$, and (ii) this fact leads to a significant redistribution of probability toward the origin of field space, which then requires an asymptotically small $Z$ factor to reestablish reasonable field averages.

\section{Numerical studies}

In a certain sense, the most basic pseudofree model lacks both the quartic coupling and the mass term. This leads to the idealized (I) model described by

$$
\begin{aligned}
S_{I}(h) \equiv & M \int \exp \left\{\Sigma h_{k} \phi_{k} a^{n} / \hbar\right. \\
& -\frac{1}{2}\left[N^{\prime 2}(q a)^{(s-1)}\right]^{-1} \sum_{k} \sum_{k^{*}}\left(\phi_{k^{*}}-\phi_{k}\right)^{2} a^{n-2} / \hbar \\
& \left.-\frac{1}{2} \hbar^{2}\left[N^{\prime 2}(q a)^{(s-1)}\right] \sum_{k} \mathcal{F}_{k}(\phi) a^{n} / \hbar\right\} \Pi_{k} d \phi_{k} .
\end{aligned}
$$

If we combine the factors of $a$, as well as transform the fields $\phi_{k}$ to remove both $\hbar$ and $q$ from the idealized action, it follows that

$$
\begin{aligned}
S_{I}(h)= & M \int \exp \left\{\sum \tilde{h}_{k} \phi_{k} a^{n}\right. \\
& \left.-\frac{1}{2} N^{\prime-2} \sum_{k} \sum_{k^{*}}\left(\phi_{k^{*}}-\phi_{k}\right)^{2}-\frac{1}{2} N^{\prime 2} \sum_{k} \mathcal{E}_{k}(\phi)\right\} \Pi_{k} d \phi_{k},
\end{aligned}
$$


where $\tilde{h}_{k} \equiv q^{(s-1) / 2} h_{k} / \hbar^{1 / 2}$ and

$$
\mathcal{E}_{k}(\phi) \equiv a^{2 s} \mathcal{F}_{k}(\phi) ;
$$

note that $\mathcal{E}_{k}(\phi)$ is dimensionless apart from any that may arise from its inverse-square field dependence. One last transformation, in which $\phi_{k} \rightarrow$ $N^{\prime} \phi_{k}$, leads to the expression

$$
\begin{aligned}
S_{I}(h)= & M \int \exp \left\{\Sigma \tilde{h}_{k} \phi_{k} N^{\prime} a^{n}\right. \\
& \left.-\frac{1}{2} \sum_{k} \sum_{k^{*}}\left(\phi_{k^{*}}-\phi_{k}\right)^{2}-\frac{1}{2} \sum_{k} \mathcal{E}_{k}(\phi)\right\} \Pi_{k} d \phi_{k} ;
\end{aligned}
$$

in the above expressions we have used the same symbol $(M)$ for the normalization factor even though it has absorbed different Jacobian factors. This last version may be useful for numerical studies of this basic pseudofree model. As argued in Sec. 5, the mass term and the quartic coupling terms can both be added by divergence-free perturbation series.

More directly, Monte Carlo studies may be made of the full nonlinear lattice theory. This can be done with a variety of rescaled field variables, but perhaps the most convenient is that of Eq. (66). In those field variables, the Euclidean lattice space probability distribution is given by

$$
\begin{aligned}
C \exp \{ & -\frac{1}{2} \sum_{k} \sum_{k^{*}}\left(\phi_{k^{*}}-\phi_{k}\right)^{2}-\frac{1}{2}\left[N^{\prime} a q^{-1}\right] m^{2} \sum_{k} \phi_{k}^{2} \\
& \left.-\left[N^{\prime 3} a q^{s-2}\right] \lambda \sum_{k} \phi_{k}^{4}-\frac{1}{2} \sum_{k} \mathcal{E}_{k}(\phi)\right\},
\end{aligned}
$$

where $C$ denotes an overall normalization factor. Traditional Monte Carlo methods may be used with this weighting to approximately determine various correlation functions. The coefficients of the $m^{2}$ and $\lambda$ terms are direct transcriptions of those determined earlier in the present section. They have been estimated to be suitable for all values of $a$ and $L$ for which $L a<\infty$. Thus they should also hold in the continuum limit with finite spatial and spacetime volumes. Any divergences that arise in the infinite spatial and spacetime volume limit should only be those that typically arise for stationary questions, such as illustrated earlier with the one-dimensional Ornstein-Uhlenbeck process; such divergences are expected and do not require any special treatment.

\section{Additional Discussion and Conclusions}

In [4] there is an extensive discussion of soluble, scalar nonrenormalizable models that are idealized versions of the relativistic model treated in the 
present paper. Such models differ from the relativistic model in that in one case all spacetime derivative terms are omitted from the classical action and in the second case all but one of the derivative terms are dropped; in this latter case, the remaining term is identified with the eventual time direction in an analog of a Wick rotation. These models have no physics and are only of academic interest. Nevertheless, from a mathematical viewpoint both of these models lead to Gaussian results if no further counter terms are introduced, and if they are studied perturbatively, they are both nonrenormalizable. Fortunately, both of these models have sufficient symmetry so that they can be rigorously solved on the basis of self-consistency without using any form of perturbation theory. One of the results for both models is that as the coupling constant of the nonlinear interaction term is reduced to zero, the theories do not return to the appropriate free theory but instead they pass continuously to an appropriate pseudofree theory. Moreover, both theories exhibit meaningful, divergence-free perturbation theories about the pseudofree theory, but definitely not about the customary free theory. These models are explicitly worked out in Chaps. 9 and 10 of ref. [4], but there is also a natural reason why such results are plausible.

Nonrenormalizable quantum field theories have exceptionally strong interaction terms. This statement can be quantified as follows: Consider the $\varphi_{n}^{p}$ relativistic scalar theory which has a free (Euclidean) action given by

$$
W=\frac{1}{2} \int\left[(\nabla \varphi)(x)^{2}+m^{2} \varphi(x)^{2}\right] d^{n} x,
$$

where $x \in \mathbb{R}^{n}$. These theories have the nonlinear interaction term

$$
V=\int \varphi(x)^{p} d^{n} x
$$

where we focus on cases where $p \in\{4,6,8, \ldots\}$. Such expressions appear in a formal functional integral such as

$$
S_{\lambda}(h)=\mathcal{N} \int e^{\int h \varphi d^{n} x-W-\lambda V} \mathcal{D} \varphi
$$

If $\lim _{\lambda \rightarrow 0} S_{\lambda}(h)=S_{0}(h)$, then the interacting theory is continuously connected to the free theory; if, instead, $\lim _{\lambda \rightarrow 0} S_{\lambda}(h)=\tilde{S}_{0}(h) \neq S_{0}(h)$, then the interacting theory is not continuously connected to the free theory, but rather it is continuously connected to a psuedofree theory. Under what conditions could this latter situation arise? 
Consider the classical Sobolev-type inequality ([4], Chap. 8) given (for $\varphi \not \equiv 0)$ by

$$
\left\{\int \varphi(x)^{p} d^{n} x\right\}^{2 / p} /\left\{\int\left[(\nabla \varphi)(x)^{2}+m^{2} \varphi(x)^{2}\right] d^{n} x\right\} \leq R
$$

as a function of the parameters $p$ and $n$. For $p \leq 2 n /(n-2)$, it follows that $R=4 / 3$; for $p>2 n /(n-2), R=\infty$ holds. This dichotomy is exactly that between perturbatively renormalizable and perturbatively nonrenormalizable models. But why should this inequality relate to renormalizability?

It is the author's long-held belief that the explanation arises from a hardcore behavior of nonrenormalizable interactions [8]. Simply stated, the interaction for such theories is so strong that a set of nonzero measure of the field histories allowed by the free theory alone is projected out when the interaction term is present. For example, $R=\infty$ means that there are fields for which $V$ is not dominated by $W$ in the same way as when $R=4 / 3$. This fact suggests that for positive coupling constant values, some of the field histories are projected out never to return as the coupling constant passes to zero. This result is the effect of a hard core at work. For the first idealized model mentioned above, the analogous ratio is $\left\{\int \varphi(x)^{4} d^{n} x\right\}^{1 / 2} /\left\{\int m^{2} \varphi(x)^{2} d^{n} x\right\}$, which for any $n \geq 1$ clearly has no finite upper bound, while for the second idealized model, the appropriate ratio reads $\left\{\int \varphi(x)^{4} d^{n} x\right\}^{1 / 2} /\left\{\int\left[\dot{\varphi}(x)^{2}+m^{2} \varphi(x)^{2}\right] d^{n} x\right\}$, which, in this case for any $n \geq 2$, has no finite upper bound. These soluble models each more singular in principle than the relativistic models - are examples of hard-core interactions that nevertheless have divergence-free perturbations about their own pseuofree model. This set of facts strongly suggests that relativistic scalar fields such as $\varphi_{n}^{4}, n \geq 5$, as we have focused on, also have a corresponding hard-core behavior.

The soluble, idealized models have formulations that involve inverse field powers; indeed, the second model, which lies closer to the relativistic models, has an inverse-square field power counter term in the lattice action itself. For both idealized models, the needed counter term was not assumed, it was derived, thanks to a large symmetry of the model. For the relativistic models, there is insufficient symmetry to derive the needed counter term, and thus the counter term must be postulated, i.e, guessed. There have been several past suggestions that have not lived up to expectations. The present paper offers one more proposal that seems to satisfy the expected requirements.

Unfortunately, the present model is not (or at least seems not) analytically tractable, probably lacking a technical means to analytically perform pertur- 
bation calculations about the pseudofree theory. Nevertheless, it would seem possible that numerical Monte Carlo calculations should be feasible. The first such calculation that should be made is a test for non-triviality that is applied to such theories by testing whether or not the Gaussian property that

$$
\left\langle\left(\Sigma_{k} h_{k} \phi_{k} a^{n}\right)^{4}\right\rangle-3\left\langle\left(\Sigma_{k} h_{k} \phi_{k} a^{n}\right)^{2}\right\rangle^{2}=0
$$

holds true for all choices of $\left\{h_{k}\right\}$ as one approaches the continuum limit; a single violation of this inequality would demonstrate that the continuum limit is not that of a free theory. In view of the connection of such full spacetime correlation functions to those on a single spatial surface, as shown in Sec. 4, it seems unlikely that (72) holds true thanks to the chosen form of the counter term; on the other hand, Sec. 4 ultimately involves inequalities which might allow (72) to sneak through.

Should the above nontriviality test prove successful for a $\varphi_{5}^{4}$ relativistic model, for example, it would be worthwhile to study various three-dimensional models such as $\varphi_{3}^{8}, \varphi_{3}^{10}$, etc. [Remark: It is noteworthy that if instead of the interaction $\lambda_{0} \phi_{n}^{4}$ discussed in the present paper we had started with $g_{0} \phi_{n}^{2 r}$, where $r \in\{3,4,5,6, \ldots\}$, then it follows that such an interaction would also possess a divergence-free perturbation expansion provided we choose

$$
g_{0}=N^{\prime(2 r-1)} a^{(s-1) r-s} g,
$$

where $g$ is the physical coupling constant. Moreover, it follows that

$$
Z^{r} g_{0}=\left[N^{\prime}(q a)^{s}\right]^{-1} g
$$

for all values of $r$, just as was the case for the mass term in (52) and for the quartic coupling in (59).] It would also be interesting to reconsider the $\varphi_{4}^{4}$

model, which, although perturbatively renormalizable, has the property of tending to a Gaussian theory in the continuum limit as shown by renormalization group studies as well as Monte Carlo studies. It is possible that a nonperturbative and nontrivial $\varphi_{4}^{4}$ model is still to be discovered.

\section{The counter term from a factor ordering ambiguity}

It is not without interest that the chosen counter term can be viewed as arising from a factor ordering ambiguity. Let $\pi_{k}$ denote the classical momentum 
conjugate to the field $\phi_{k}$ for all $k$ in a spatial slice. The classical Hamiltonian reads

$$
H=\frac{1}{2} \sum_{k}^{\prime} \pi_{k}^{2} a^{s}+V_{0}(\phi)=\frac{1}{2} \sum_{k}^{\prime} D \pi_{k} D^{-2} \pi_{k} D a^{s}+V_{0}(\phi),
$$

where $D=D(\phi) \equiv \Pi_{k}^{\prime}\left[\Sigma_{l}^{\prime} J_{k, l} \phi_{l}^{2}\right]^{\left(N^{\prime}-1\right) / 4 N^{\prime}}$. Passing to the quantum theory leads to the Hamiltonian operator [c.f., (11)]

$$
\begin{aligned}
\mathcal{H} & =-\frac{\hbar^{2}}{2} a^{-s} \sum_{k}^{\prime} D \frac{\partial}{\partial \phi_{k}} D^{-2} \frac{\partial}{\partial \phi_{k}} D+\mathcal{V}_{0}(\phi) \\
& =-\frac{\hbar^{2}}{2} a^{-s} \sum_{k}^{\prime} \frac{\partial^{2}}{\partial \phi_{k}^{2}}+\mathcal{V}_{0}(\phi)+\frac{\hbar^{2}}{2} \sum_{k}^{\prime} \mathcal{F}_{k}(\phi) a^{s}
\end{aligned}
$$

\section{Classical limit}

When dealing with a nonrenormalizable $\varphi_{n}^{4}$ theory, we argued in Sec. 1 against choosing either no counter terms or those counter terms suggested by a regularized perturbation analysis about the free theory. This was due, in part, to the fact that such theories tend not to have the correct classical limit, namely, the original nonlinear classical theory one started with. That property is clear in the case of no counter terms, which leads to a free quantum theory, and it is effective as well when perturbative counter terms are considered because in the latter case there is no complete and well-defined quantum theory for which the classical limit can be studied.

One strong test of whether or not the ideas in this paper have some validity would be to try to take the classical limit and confirm that the expected nonlinear relativistic theory emerges. The study of this question first requires having some control on the continuum limit, but in support of its possible realization we note that the second idealized model treated in Chap. 10 of [4], namely, the model including the time derivative of the field, has been shown to indeed have the correct classical limit for the idealized but nonlinearly interacting model in question. It is possible that similar techniques may be used to show that the presently proposed quantization scheme for nonrenormalizable $\varphi_{n}^{4}$ models has a classical limit that agrees with the original nonlinear classical theory.

Even if our proposal leads to a nontrivial quantum theory, and even if that quantum theory exhibits a correct classical limit, the question may arise whether this proposal for quantization is the "correct" quantization 
procedure. As in any quantization procedure, where one starts from a theory with $\hbar=0$ and constructs a theory with $\hbar>0$, there is a great deal of latitude in the result. Nevertheless, in the absence of any other satisfactory proposal to deal with nonrenormalizable theories, one might look favorably on a model that offers more than was previously available.

\section{References}

[1] M. Aizenman, "Proof of the Triviality of $\varphi_{d}^{4}$ Field Theory and Some Mean-Field Features of Ising Models for $d>4$ ", Phys. Rev. Lett. 47, 1-4, E-886 (1981).

[2] J. Fröhlich, "On the Triviality of $\lambda \varphi_{d}^{4}$ Theories and the Approach to the Critical Point in $d \geq 4$ Dimensions", Nuclear Physics B 200, 281296 (1982). See also: R. Fernández, J. Fröhlich, and A. Sokal, Random Walks, Critical Phenomena, and Triviality in Quantum Field Theory, (Springer-Verlag, New York, 1992).

[3] J.R. Klauder, "A New Approach to Nonrenormalizable Models", Ann. Phys. 322, 2569-2602 (2007).

[4] J.R. Klauder, Beyond Conventional Quantization, (Cambridge University Press, Cambridge, 2000 \& 2005).

[5] J.R. Klauder, "Poisson Distributions for Sharp-Time Fields: Antidote for Triviality", hep-th/9511202, pp 22-28.

[6] J.R. Klauder, "Isolation and Expulsion of Divergences in Quantum Field Theory", Int. J. Mod. Phys. B 10, 1473-1483 (1996).

[7] T. Hida and M. Hitsuda, Gaussian Processes, (American Mathematical Society, Providence, 2007).

[8] J.R. Klauder, "Field Structure through Model Studies: Aspects of Nonenormalizable Theories", Acta. Phys. Austr. Suppl. XI, 341-387 (1973). 